\title{
Microvascular and Macrovascular Complications In Type 2 Diabetes Milletus
}

\author{
Prakash Ajmera ${ }^{\circledR 1}$, P Sailaja ${ }^{\circledR 2}$, P Raghu Ramulu ${ }^{\circ} 3$ \\ ${ }^{1}$ Assistant Professor, Department of General Medicine, Bhaskar Medical College \& Bhaskar General Hospital, Bhaskar Nagar, Moinabad, Hyderabad, India, ${ }^{2}$ Assistant \\ professor, Department of Gynaecology, Bhaskar Medical College \& Bhaskar General Hospital, Bhaskar Nagar, Moinabad, Hyderabad, India, ${ }^{3}$ Professor and Head, \\ Department of General Medicine, Bhaskar Medical College \& Bhaskar General Hospital, Bhaskar Nagar, Moinabad, Hyderabad, India.
}

\section{Abstract}

Background: Long-term damage and malfunction of various organ systems can be linked to diabetes and its related complications. The objective is to evaluate the microvascular and macrovascular complications of Type 2 Diabetes Mellitus. Subjects and Methods: Patients with confirmed diabetes attending OPD were included. A detailed history is recorded for each individual case with diabetes mellitus, obesity, symptoms and family history with individuals suffering from diabetes mellitus. The physical examination was done for sensory and motor signs. Prevalence rates were typically calculated and standardized for age and sex for microvascular and macrovascular complications at baseline. Results: The overall number of patients examined was 100, of which 54\% were male and $46 \%$ were female. The mean age of the patients in the sample was 53 years. The prevalence of diabetes increased with age. The prevalence of diabetes in elderly patients was maximum for 61 to 70 years of age. 29 percent of patients have a positive history of diabetes in the family. $13 \%$ of patients were smokers and all of them were males. The risk factor - Hypertension $-19 \%$, Obesity $-25 \%$ and hypercholesterolemia $-43 \%$ were present at the time of diagnosis. Conclusion: The general risk of microvascular and macrovascular problems is severe in patients with type 2 diabetes that are relatively early in the disease phase. Such findings indicate that the early risk factor may be actively changed, especially in regions with a high prevalence of complications. There are important correlations between predominant diabetes and the rising circumference and body mass index. Coronary artery disease, nephropathy and retinopathy have become particularly prevalent.

Keywords: Microvascular, Macrovascular, Diabetes Mellitus, Chronic Kidney Disease

Corresponding Author: P Raghu Ramulu, Professor and Head, Department of General Medicine, Bhaskar Medical College \& Bhaskar General Hospital, Bhaskar Nagar, Moinabad, Hyderabad, India.

E-mail: drpraghuramulu364@gmail.com

Received: 30 June 2020

Revised: 14 August 2020

Accepted: 22 August 2020

Published: 26 December 2020

\section{Introduction}

Diabetes Mellitus is a group of chronic metabolic disorders that share the phenotype of hyperglycemia. There are many different forms of Diabetes Mellitus caused by diverse genetic, environmental and behavioral factors. Diabetes mellitus is classified into two group's type 1 and type 2. Autoimmune Beta-cell death leads to type 1 diabetes. Type 2 Diabetes Mellitus is a heterogeneous collection of conditions of differing degrees of insulin tolerance, low insulin secretion and elevated glucose output. The chronic condition of DM involves a variety of organ systems and contributes to the vast majority of the disease's morbidity and mortality. Diabetes Mellitus's vascular complications are divided into microvascular (neuropathic retinopathy, nephropathy) complications and Macrovascular (cerebrovascular [CVD], coronary artery disorders [CAD], peripheral artery [PVD]) complications. ${ }^{[1]}$
Chronic hyperglycemia occurs in micro-vascular disorders in both type 1 and types 2 DM. ${ }^{[2]}$ Since T2DM also has a long hyperglycemic asymptomatic period, many individuals with T2DM have diagnostic difficulties. There is less definitive evidence that chronic hyperglycemia plays a causal role in macrovascular complications. Cardiac diseases and death rates are also 2-4 times higher in individuals with T2DM. Additional factors such as dyslipidemia and high blood pressure are also critical for macrovascular complications. Microvascular complications of diabetes are long-termcomplications affecting smaller blood vessels. Usually retinopathy, nephropathy and neuropathy are among them. Two main types are retinopathy: proliferative retinopathy and non-proliferative retinopathy. Chronic proteinuria in diabetic nephropathy. This is marked by a progressive deterioration in renal function which results in renal disease. It will proceed in overt nephropathy. Neuropa- 
thy is a heterogeneous condition associated with nerve pathology. Sensory, motor, focal, diffuse and autonomous neuropathy categorizes the nerve condition. Macrovascular diabetes disorders are predominantly of coronary and peripheral arteries and cerebrovasculature. The leading cause of morbidity and death of diabetes patients is cardiovascular disease (CVD). Early macrovascular condition is associated with atherosclerotic vascular plaque. Late stages of the macrovascular disease involve complete obstruction of these vessels. This can increase the risks of myocardia infarction (MI), claudication, stroke, and gangrene. ${ }^{[3]}$

\section{Subjects and Methods}

Sampling Methods: Consecutive sampling. Approval was taken from the Institutional Ethics Committee prior to the commencement of the study. The study was a hospital-based retrospective study.

\section{Inclusion Criteria}

T2DM patients over 35 years old were evaluated in the outpatient department for risk factors, micro and macrovascular complications for which they were categorized according to the duration of diabetes based on below duration

- Newly Diagnosed

- Diabetes Mellitus forless than 5 Years

- Diabetes Mellitusfor 5 to 10 Years.

- Diabetes Mellitusfor more than 10 Years.

A detailed history is recorded for each individual case with diabetes mellitus, obesity, symptoms and family history with individuals suffering from diabetes mellitus.The physical examination was done for sensory and motor signs.

\section{Exclusion Criteria}

Those not willing to give consent or co-operate include T1DM, known diabetes mellitus and getting treated, pregnant ladies and people with severe illness were excluded from our study.

\section{Statistical Analysis}

Data in statistical tables have been presented. The statistical research was done using SPSS version 20.

\section{Results \& Discussion}

The overall number of examined patients was 100 , of which $54 \%$ were male and $46 \%$ were female. The mean patient age was 53 years. These results were similar to Raheja et al, ${ }^{[4]}$ research, which reported the mean age of 53.3 and further analysis in the National Diabetic Rural Survey, which indicated a mean age of 52.3-years. ${ }^{[5]}$ With age, diabetes incidence is increased. In elderly patients in the sample, the incidence of diabetes is 22 percent and in the elderly, a maximum number of cases were seen in the 61 to 70 years of age group. This result is in line with the Jain A research. ${ }^{[6]} \mathrm{Of}$ the 100 cases, $30 \%$ have a clear history of diabetes in their families. Positive family history with type 2 diabetes is closely related in several Indian studies. These results are in line with the Shah et al, ${ }^{[7]}$ research which showed a positive family history of $24.9 \%$ and that of Ramachandran et al, ${ }^{[8]}$ for which positive family history has been closely associated with T2DM.

\section{Microvascular Complications}

The most prevalent microvascular disorder is diabetic retinopathy seen in $34 \%$ of the cases. $74 \%$ of the patients had non-proliferative retinopathy. Based on diabetes duration, the incidence of retinopathy increases. This research is in line with Darivemula $\mathrm{S}$ et al, ${ }^{\left[{ }^{[]}\right.}$study of Urban South Indian population which showed Retinopathy in $34 \%$ of the study sample, and in North Indian study by Barman et al. ${ }^{[10]}$ Observed Retinopathy in $29 \%$ of the study sample. 35 percent of research subjects showed CKD in diabetes. In stage 2 the total number of patients with CKD was $43 \%$. Proteinuria was seen in $16 \%$ of them. In 21 percent of patients, diabetic neuropathy was predominant. This result corresponds to the research carried out by Darivemula S et al which showed 19 percent of patients with neuropathy. ${ }^{[9]}$ A important result in the study at the time of diabetes mellitus diagnosis was retinopathy (34\%), neuropathy (22\%) and nephropathy (31\%).

\section{Macrovascular Complications}

The most prevalent macrovascular complication in the study is coronary artery disease seen in $35 \%$ of the cases. The current study reported asymptomatic Coronary Artery Disease in 45 percent of patients. Diabetes Mellitus age was $<5$ years for 42 percent of patients with Coronary Artery Disease. Nearly 5 percent of patients in the sample suffered from peripheral vascular disease. This is related to the 4 percent as seen in the analysis by Barman et al. ${ }^{[10]}$

Almost $11 \%$ of the study patients were diagnosed with the cerebrovascular disorder. This is in line with the 2005 CDCNational Diabetes Surveillance System (US) international study which showed a prevalence of 9 percent of strokes in patients with T2DM. ${ }^{[1]}$

\section{Conclusion}

The prevalence of diabetes and increased waist circumference and BMI is very closely related. Coronary heart disease, nephropathy and retinopathy have become particularly prevalent. This research emphasizes the need for early diagnosis, monitoring, and awareness programs in order to reduce the burden of complication management. Effective blood glucose regulation decreases microvascular disorder and should 
Table 1: Age, Sex \& Risk Factors Distribution

\begin{tabular}{llll}
\hline Age (In Yrs) & Male & Female & Total n= 100 \\
34 to 40 & 4 & 3 & $7(7 \%)$ \\
41 to 50 & 15 & 13 & $28(28 \%)$ \\
51 to 60 & 21 & 22 & $43(43 \%)$ \\
61 to 70 & 11 & 7 & $18(18 \%)$ \\
$>70$ & 2 & 2 & $4(4 \%)$ \\
Risk Factors & & 7 & $19(19 \%)$ \\
Hypertension & 12 & 15 & $25(25 \%)$ \\
Obesity & 10 & - & $13(13 \%)$ \\
Smoking & 13 & 24 & $43(43 \%)$ \\
\hline Hypercholestrolemia & 19 & & \\
\hline
\end{tabular}

Table 2: Various Microvascular Complications and Duration of Diabetes Mellitus in years

\begin{tabular}{|c|c|c|c|c|c|}
\hline $\begin{array}{l}\text { Microvascular } \\
\text { Complication }\end{array}$ & $\begin{array}{l}\text { Newly Diagnosed } \\
(n=31)\end{array}$ & $<5 \operatorname{yrs}(n=33)$ & 5 to 10 yrs $(n=23)$ & $10 \operatorname{yrs}(n=13)$ & Total $(n=100)$ \\
\hline Retinopathy & $13(38.23 \%)$ & $9(26.47 \%)$ & $7(20.58 \%)$ & $5(14.70 \%)$ & $34(34 \%)$ \\
\hline Nephropathy & $6(19.35 \%)$ & $13(41.93 \%)$ & $10(32.25 \%)$ & $2(6.45 \%)$ & $31(31 \%)$ \\
\hline
\end{tabular}

\section{Table 3: Types of Diabetic Retinopathy}

\begin{tabular}{ll}
\hline Types & Total $(\mathbf{n}=\mathbf{3 4})$ \\
Maculopathy & $4(11.76 \%)$ \\
Proliferative retinopathy & $5(14.70 \%)$ \\
Non - proliferative retinopathy & $25(73.52 \%)$ \\
\hline
\end{tabular}

Table 4: Stages of CKD in Diabetes

\begin{tabular}{lll}
\hline GFR & Stages of CKD & Total $\mathbf{n}=\mathbf{3 5}$ \\
90 to 120 & Stage 1 & $9(25.71 \%)$ \\
60 to 89 & Stage 2 & $15(42.85 \%)$ \\
30 to 59 & Stage 3 & $11(31.42 \%)$ \\
$<30$ & Stage 4 \& 5 & - \\
\hline
\end{tabular}

\section{Table 5: Coronary Artery Disease \& Diabetes Mellitus}

\begin{tabular}{ll}
\hline Duration & Total $\mathbf{n}=\mathbf{3 1}$ \\
Newly diagnosed & $9(29.03 \%)$ \\
$<5$ Yrs & $13(41.93 \%)$ \\
5 to 10 Yrs & $6(19.35 \%)$ \\
$>10$ Yrs & $3(9.67 \%)$ \\
\hline
\end{tabular}

\section{Table 6: Macrovascular Complications \& Diabetes Mellitus}

\begin{tabular}{ll}
\hline Complications & Total $\mathbf{n}=\mathbf{1 0 0}(\mathbf{1 0 0} \%)$ \\
Coronary Artery Disease & $35(35 \%)$ \\
Peripheral VascularDisease & $5(5 \%)$ \\
Cerebro Vascular Disease & $11(11 \%)$ \\
\hline
\end{tabular}


Table 7: Fasting Blood Sugar Level \& Diabetes Mellitus

\begin{tabular}{llllll}
$\begin{array}{l}\text { Fasting Blood Sug- } \\
\text { armg/dL }\end{array}$ & Newly diagnosed & $<\mathbf{5}$ yrs & $\mathbf{5}$ to $\mathbf{1 0}$ yrs & $\mathbf{1 0}$ yrs & Total $\mathbf{n}=\mathbf{1 0 0}$ \\
\hline 126 to 200 & 11 & 22 & 10 & 2 & $45(45 \%)$ \\
201 to 300 & 8 & 10 & 3 & 1 & $22(22 \%)$ \\
$>300$ & 4 & 7 & 2 & - & $13(13 \%)$ \\
\hline
\end{tabular}

be enforced early and sustained for a sufficient amount of time. In order to avoid macrovascular disease in addition to glycemic regulation, proper blood pressure management and dyslipidemia are highly necessary. Patients tend to be more vulnerable to accelerated atherosclerosis and premature death in microvascular complications. Clearer insights into discrepancies in response to therapeutic treatments could lead not only to microvascular but also to macrovascular complications to help control and improve T2DM outcomes.

\section{References}

1. Orasanu G, Plutzky J. The Pathologic Continuum of Diabetic Vascular Disease. J Am Coll Cardiol. 2009;53(5):S35-S42. Available from: https://dx.doi.org/10.1016/j.jacc.2008.09.055.

2. Standards of medical care in diabetes-2016: Summary of revisions. Diabetes Care. 2016;39(S1):S4-S5. Available from: https://doi.org/10.2337/dc16-S003.

3. Fuller JH, , Stevens LK, Wang SL. Risk factors for cardiovascular mortality and morbidity: The WHO multinational study of vascular disease in diabetes. Diabetologia. 2001;44(S2):S54S64. Available from: https://dx.doi.org/10.1007/p100002940.

4. Raheja BS. Diabcare Asia: India Study: Diabetes Care in India - Current Status. 2001;49:717-722.

5. Rao PV, Shyam C. Reflectometry in epidemiological studies for diabetes mellitus - Indian. J Endocrinol Metab. 1997;1:1618.

6. Jain A, Paranjape S. Prevalence of type 2 diabetes mellitus in elderly in a primary care facility: An ideal facility. Indian J Endocrinol Metab. 2013;17(Supp11):S318-S322. Available from: https://dx.doi.org/10.4103/2230-8210.119647.
7. Shah S, Kumar. High Prevalence of Type 2 Diabetes in Urban Population in North Eastern India. Type 2 Diabetes - Intl. J DiabDev Countries. 1999;19:144-147.

8. Ramchandran A, Snehalatha C, Satyavani K, Latha E, Sasikala R, Vijay V. Prevalence of vascular complications and their risk factors in type 2 diabetes. J Assoc Physicians India. 1999;47(12):1152-1158.

9. Darivemula S, Nagoor K, Patan SK, Reddy NB, Deepthi CS, Chittooru CS. Prevalence and Its Associated Determinants of Diabetic Peripheral Neuropathy (DPN) in Individuals Having Type-2 Diabetes Mellitus in Rural South India. Indian J Community Med. 2019;44(2):88-91.

10. Barman KK, Premalatha G, Mohan V. Tropical chronic pancreatitis. Postgraduate Med J. 2003;79:606-615.

11. Deshpande AD, Harris-Hayes M, Schootman M. Epidemiology of Diabetes and Diabetes-Related Complications. Phys Ther. 2008;88(11):1254-1264. Available from: https://dx.doi.org/10. 2522/ptj.20080020.

Copyright: (C) the author(s), 2020. It is an open-access article distributed under the terms of the Creative Commons Attribution License (CC BY 4.0), which permits authors to retain ownership of the copyright for their content, and allow anyone to download, reuse, reprint, modify, distribute and/or copy the content as long as the original authors and source are cited.

How to cite this article: Ajmera P, Sailaja P, Ramulu PR. Microvascular and Macrovascular Complications In Type 2 Diabetes Milletus. Acad. J Med. 2020;3(2):16-19.

DOI: dx.doi.org/10.47008/ajm.2020.3.2.4

Source of Support: Nil, Conflict of Interest: None declared. 